\title{
Robotic surgery can extend surgical indication in patients with lung cancer and impaired function
}

\author{
Giulia Veronesi, Pietro Bruschini, Pierluigi Novellis \\ Division of Thoracic and General Surgery, Humanitas Clinical and Research Center, Rozzano, Milan, Italy \\ Correspondence to: Giulia Veronesi. Humanitas Clinical and Research Center, Thoracic Surgery Division, Via Alessandro Manzoni 56, 20089 Rozzano, \\ Milano, Italy. Email: giulia.veronesi@humanitas.it; giulia.veronesi@cancercenter.humanitas.it. \\ Provenance: This is an invited article commissioned by the Section Editor Laura Chiara Guglielmetti (Cantonal Hospital Winterthur, Kantonsspital \\ Winterthur, Winterthur, Switzerland). \\ Comment on: Kneuertz PJ, D'Souza DM, Moffatt-Bruce SD, et al. Robotic lobectomy has the greatest benefit in patients with marginal pulmonary \\ function. J Cardiothorac Surg 2018;13:56.
}

Submitted Aug 22, 2019. Accepted for publication Oct 07, 2019.

doi: $10.21037 /$ jtd.2019.10.14

View this article at: http://dx.doi.org/10.21037/jtd.2019.10.14

The topic of the careful assessment of respiratory function has always been of great importance in the evaluation of the risk of a surgical patient (1-4) but it is even more relevant today since non-surgical local treatment using stereotactic radiotherapy is emerging as a valid alternative to surgery in patients with increased surgical risks due to impaired cardio respiratory function (5).

During the last 30 years, a number of papers have been published addressing the role of FEV1 in defining the risk of perioperative morbidity and mortality after lung resection $(1-4,6,7)$. Subsequent studies suggested not to exclude patients from surgical indication on the basis of the ppoFEV1 due to limits of this variable in predicting the surgical risks in term of pulmonary function $(6,7)$. There is increasing evidence for the DLCO as an additional parameter of risk in pulmonary resection $(2,8,9)$. Liptay et al. (8) examined early and late prognostic significance of DLCO and FEV 1 in patients undergoing lung cancer surgery and found that DLCO $<40 \%$ was the best predictor of decreased survival from non-oncological causes in a group of patients with stage I lung cancer (HR 0.96, $\mathrm{P}<0.0001$ ). While Ferguson et al. on 1,008 patients submitted to major lung resections, showed that the ppoDLCO was a significant predictor of pulmonary complications and mortality in both, patients with COPD and normal function (2), confirming the added value of the ppoDLCO for the risk stratification. At this regard Kobayashi et al. suggest that the 3D-CT volumetry was a better tool than the segment-counting method to predict the residual pulmonary function, in particular in patients with COPD, optimising the selection of appropriate surgical candidates among patients with limited lung function (2). Nevertheless, most recently the importance of the maximal oxygen consumption measured in $\mathrm{mL} / \mathrm{kg} / \mathrm{min}\left(\mathrm{VO}_{2} \max \right)$ as absolute value, was demonstrated as the optimal parameter in order to quantify the risk for lung surgery $(10,11)$.

Regarding the correlation between pulmonary function, type of approach and complications, the notion of traditional surgeons is that a patient with limited pulmonary function should be operated using an "open approach" because it is "faster" than video assisted thoracoscopic surgery (VATS). This conviction was probably based on studies that showed how the duration of one lung ventilation (OLV) was one of the risk factors of ALI/ARDS and other respiratory complications in open surgery $(12,13)$. This concept, true for open surgery, was not confirmed for minimally invasive surgery. Another conviction, which was never confirmed to justify the open approach, was the low tolerability to OLV of COPD patients, required during VATS procedures.

Nevertheless many improvements have been made during the last decades in strategies to treat, prevent and reduce ALI/ARDS during OLV including lung protection from barotrauma and volutrauma (12) independent of the approach.

Undoubtedly, minimally invasive surgery has been 
shown to be related to less trauma to the patient, less manipulation of the lung and consequently fewer postoperative complications than open surgery (14). It is also true that VATS and robotic assisted videothoracoscopic surgery (RATS) more than open surgery, mandates the use of OLV with a dual lumen endotracheal tube, thus necessitating the use of a lung protective strategy (13). The new experimental procedures that use anesthesia with an awake and non-intubated patient for VATS approach are an exception, this type of anesthesia aims at reducing the potential complications of tracheal intubation, such as airway trauma due to intubation, lesion ventilation induced pulmonary and postoperative nausea and vomiting (15), however important issues should be solved before confirming the validity and feasibility of these procedures. The experience of Algahmdi et al. for example, showed that the perioperative outcomes for the nonintubated VATS lobectomy were similar to the intubated ones but the number of lymph nodes removed were lower in the non-intubated VATS group, thus further prospective studies are needed to assess the benefits of this procedure (16).

Since 2010, Berry et al. showed in a retrospective observational study that the FEV1 was significantly correlated to respiratory morbidity for the patients treated with open approach but not for the ones treated with a VATS approach (3). They analysed a cohort of 340 patients with a FEV1 or a DLCO $<60 \%$ submitted to lung lobectomy by open or VATS approach. The overall morbidity rate was $48 \%$ and the mortality rate $5 \%$, the level of FEV1 was inversely correlated with pulmonary complications within the thoracotomy group but not for the VATS group.

In this scenario, the paper by Kneuertz (17) analysed a monoistitutional retrospective cohort of 599 patients undergoing lobectomy by RATS $(\mathrm{n}=287)$ or by open approach $(\mathrm{n}=312)$ including 189 high risk subjects and showed that overall pulmonary complications were significantly lower following RATS as compared with open lobectomy ( $22 \% v s .32 \%)$ but the difference in pulmonary complications was even higher in the high-risk group (28\% vs. $45 \%, \mathrm{P}=0.02$ ) with a significantly lower duration of postoperative stay in the RATS versus open group (4 vs. 8 days; $\mathrm{P}<0.001)$. Despite, some limitations of the paper, such as the retrospective nature of the study, the absence of data on extension of resected lymph nodes in the two groups, lack of comparison between VATS and RATS and no surgical details on the open approach adopted versus (lateral muscle sparing versus posterolateral thoracotomy), the large number of cases allowed for a proper multivariable analysis. Thus the work of Kneuertz et al. has further underlined how in patients with limited function the robotic approach brings most benefits than open surgery (17).

Already in 2010 our group (18) compared RATS lobectomy versus propensity matched lateral muscle sparing thoracotomy: we found similar complication rate and similar number of lymph nodes in the two groups but shorter postoperative stay after RATS lobectomy despite the robotic cases included also the learning curve. Better results were obtained in 2011 by Cerfolio et al. (19) that reported their analysis of over one hundred patients undergoing RATS lobectomy versus a nerve-sparing thoracotomy and found that RATS had significantly lower postoperative morbidity. Subsequently different papers, including the metanalysis of the Collaborative Research (CORE) Group, the paper by Kent et al., and the paper by NG et al. (20-24) found fewer complications after RATS than open surgery found that RATS was associated with significantly reduced mortality, postoperative stay and complications.

When comparing RATS versus VATS most papers demonstrated similar perioperative outcome, including conversion rate and morbidity $(21,22)$. One metanalysis involving 58,683 VATS and 3,375 RATS resection (23) showed a reduced postoperative mortality rate after RATS versus VATS resections (HR 0.52, 0.29-093) while other analysis $(24,25)$ showed an improved lymph node dissection associated to RATS versus VATS. For this last reason, RATS approach can also be safely used for the treatment of selected locally advanced disease as allows an adequate radical treatment. Thanks to this innovative technology an increased number of lung cancer patients can benefit of a minimally invasive treatment, in particular those requiring complex procedures after induction therapy (26).

This concept paves the way to a number of considerations for example when assessing the risk benefit balance between surgery and SBRT (stereotactic body radiotherapy) in the treatment of early stage lung cancer.

The recently published recommendations on the use of SBRT versus surgery in early-stage 1 lung cancer from ASCO/ASRO society (27) indicates that for patients fit for surgery, SBRT is not contemplated outside clinical trials while for high-risk patients, SBRT can be considered after adequate discussion within the MDT, provided patients are informed of both decreased treatment risks and the unknown long-term outcomes; in addition SBRT should be carefully considered for centrally located tumors, due to the increased risk of toxicity. In addition it is recommended that 
the high-risk setting is defined by FEV1 and DLco $<50 \%$, or when there are additional risk factors, such as advanced age, impaired pulmonary function, pulmonary hypertension, or poor left-ventricle function (27). Similar cut-off of pulmonary function has been confirmed to be associated to increased complications in our recent analysis on the impact of pulmonary function on pulmonary complications after robotic thoracic surgery (28).

To aid selection of candidates with borderline cardiopulmonary function, Brunelli et al. developed a model of 30 days mortality and cardiopulmonary morbidity from retrospective analysis of data of more than 47 thousand patients recorded in the ESTS database. The Aggregate risk score (Aggregate EuroLung1) was developed based on proportional weighting of the coefficients, assigning 1 point to CKD (Kidney diseases comorbidities); 2 point to CAD (cardiac disease) and CVD (cerebrovascular diseases); 3 points to age $>65$, sex male, thoracotomy, extended resections and ppoFEV $1<70 \%$. The points were summed to generate an aggregate score in 6 classes of incremental morbidity risk. Thoracotomy approach was one of the variables related to increased risk with 3 points (29).

According to the findings of Kneuertz (17) and other previous works the minimally invasive approaches should be considered in comparative trials of surgery versus SBRT treatment as it is associated to reduced complications (30). Among them, RATS may represent the best minimally invasive surgical approach of the future in relation to the improved mediastinal and hilar lymph node dissection obtained $(24,25)$, the indisputable technical advantages including better ergonomics and optimal 3D view and the possibility to easily perform complex interventions such as difficult anatomical segmentectomies using indocyanine green (31) for very early stages, thus sparing useful lung parenchima.

Beyond minimally invasive incisions, to enhance surgical outcome, novel protocols assess pre-operative frailty measures, which is a more significant marker of surgical risk than age and COPD. A number of investigators have demonstrated the possibility to identify in a preoperative setting frail patients using frailty scores $(32,33)$ and support the opportunity to introduce routine "pre-habilitation" to reduce operative morbidity in frail patients. Recent evidence suggests that 2-4 weeks high-intensity interval training (HIIT) provides a significant rehabilitation for high-risk frail patients $(34,35)$.

To conclude surgical resection provides the highest chance of cure in patients with early stage non-small cell lung carcinoma. However, in COPD patients, this approach can bear an increased risk of mortality and morbidity, thus requiring an accurate pre-operatory evaluation of the surgical risk and a frailty score, high intensity interval prehabilitation in frail patients, discussion in multidisciplinary tumor board for indication to appropriate local treatment, in case of surgery selection of the less invasive surgical approaches able to reduce the surgical risk maintaining the radicality of treatment, accurate intraoperative lung protective OLV measures and intense postoperative rehabilitation.

\section{Acknowledgments}

The Umberto Veronesi Foundation is gratefully acknowledged for fellowship to Pietro Bruschini.

\section{Footnote}

Conflicts of Interest: Giulia Veronesi has received honoraria from AbMedica SpA, Medtronic and Verb Medical. The other authors have no conflicts of interest to declare.

Ethical Statement: The author is accountable for all aspects of the work in ensuring that questions related to the accuracy or integrity of any part of the work are appropriately investigated and resolved.

\section{References}

1. Lim E, Baldwin D, Beckles M, et al. Guidelines on the radical management of patients with lung cancer. Thorax 2010;65 Suppl 3:iii1-27.

2. Ferguson MK, Little L, Rizzo L, et al. Diffusing capacity predicts morbidity and mortality after pulmonary resection. J Thorac Cardiovasc Surg 1988;96:894-900.

3. Berry MF, Villamizar-Ortiz NR, Tong BC, et al. Pulmonary function tests do not predict pulmonary complications after thoracoscopic lobectomy. Ann Thorac Surg 2010;89:1044-51; discussion 1051-2.

4. Ferguson MK, Reeder LB, Mick R. Optimizing selection of patients for major lung resection. J Thorac Cardiovasc Surg 1995;109:275-81; discussion 281-3.

5. Wang S, Wang X, Zhou Q, et al. Stereotactic ablative radiotherapy versus lobectomy for stage I non-small cell lung cancer: A systematic review. Thorac cancer 2018;9:337-47.

6. Brunelli A, Al Refai M, Monteverde M, et al. Predictors 
of early morbidity after major lung resection in patients with and without airflow limitation. Ann Thorac Surg 2002;74:999-1003.

7. Sekine Y, Iwata T, Chiyo M, et al. Minimal alteration of pulmonary function after lobectomy in lung cancer patients with chronic obstructive pulmonary disease. Ann Thorac Surg 2003;76:356-61; discussion 362.

8. Liptay MJ, Basu S, Hoaglin MC, et al. Diffusion lung capacity for carbon monoxide (DLCO) is an independent prognostic factor for long-term survival after curative lung resection for cancer. J Surg Oncol 2009;100:703-7.

9. Brunelli A, Refai MA, Salati M, et al. Carbon monoxide lung diffusion capacity improves risk stratification in patients without airflow limitation: evidence for systematic measurement before lung resection. Eur J Cardiothorac Surg 2006;29:567-70.

10. Brunelli A, Belardinelli R, Refai M, et al. Peak oxygen consumption during cardiopulmonary exercise test improves risk stratification in candidates to major lung resection. Chest 2009;135:1260-7.

11. Licker M, Schnyder JM, Frey JG, et al. Impact of aerobic exercise capacity and procedure-related factors in lung cancer surgery. Eur Respir J 2011;37:1189-98.

12. Grichnik KP, D’Amico TA. Acute lung injury and acute respiratory distress syndrome after pulmonary resection. Semin Cardiothorac Vasc Anesth 2004;8:317-34.

13. Williams EA, Evans TW, Goldstraw P. Acute lung injury following lung resection: is one lung anaesthesia to blame? Thorax 1996;51:114-6.

14. Ujiie H, Gregor A, Yasufuku K. Minimally invasive surgical approaches for lung cancer. Expert Rev Respir Med 2019;13:571-8.

15. Gonzalez-Rivas D, Bonome C, Fieira E, et al. Nonintubated video-assisted thoracoscopic lung resections: the future of thoracic surgery? Eur J Cardiothorac Surg 2016;49:721-31.

16. AlGhamdi ZM, Lynhiavu L, Moon YK, et al. Comparison of non-intubated versus intubated video-assisted thoracoscopic lobectomy for lung cancer. J Thorac Dis 2018;10:4236-43.

17. Kneuertz PJ, D'Souza DM, Moffatt-Bruce SD, et al. Robotic lobectomy has the greatest benefit in patients with marginal pulmonary function. J Cardiothorac Surg 2018;13:56.

18. Veronesi G, Galetta D, Maisonneuve P, et al. Four-arm robotic lobectomy for the treatment of early-stage lung cancer. J Thorac Cardiovasc Surg 2010;140:19-25.

19. Cerfolio RJ, Bryant AS, Skylizard L, et al. Initial consecutive experience of completely portal robotic pulmonary resection with 4 arms. J Thorac Cardiovasc Surg 2011;142:740-6.

20. Cao C, Manganas C, Ang SC, et al. A systematic review and meta-analysis on pulmonary resections by robotic video-assisted thoracic surgery. Ann Cardiothorac Surg 2012;1:3-10.

21. Kent M, Wang T, Whyte R, et al. Open, video-assisted thoracic surgery, and robotic lobectomy: review of a national database. Ann Thorac Surg 2014;97:236-42; discussion 242-4.

22. Ng CSH, MacDonald JK, Gilbert S, et al. Optimal Approach to Lobectomy for Non-Small Cell Lung Cancer: Systemic Review and Meta-Analysis. Innovations (Phila) 2019;14:90-116.

23. Emmert A, Straube C, Buentzel J, et al. Robotic versus thoracoscopic lung resection: A systematic review and meta-analysis. Medicine (Baltimore) 2017;96:e7633.

24. Novellis P, Bottoni E, Voulaz E, et al. Robotic surgery, video-assisted thoracic surgery, and open surgery for early stage lung cancer: comparison of costs and outcomes at a single institute. J Thorac Dis 2018;10:790-8.

25. Toker A, Özyurtkan MO, Demirhan Ö, et al. Lymph Node Dissection in Surgery for Lung Cancer: Comparison of Open vs. Video-Assisted vs. Robotic-Assisted Approaches. Ann Thorac Cardiovasc Surg 2016;22:284-90.

26. Veronesi G, Park B, Cerfolio R, et al Robotic resection of Stage III lung cancer: an international retrospective study. Eur J Cardiothorac Surg 2018;54:912-9.

27. Schneider BJ, Daly ME, Kennedy EB, et al. Stereotactic Body Radiotherapy for Early-Stage Non-Small-Cell Lung Cancer: American Society of Clinical Oncology Endorsement of the American Society for Radiation Oncology Evidence-Based Guideline. J Clin Oncol 2018;36:710-9.

28. Cao C, Louie BE, Melfi F, et al. Impact of pulmonary function on pulmonary complications a fter roboticassisted thoracoscopic lobectomy. Eur J Cardiothorac Surg 2019. [Epub ahead of print].

29. Brunelli A, Salati M, Rocco G, et al. European risk models for morbidity (EuroLung1) and mortality (EuroLung2) to predict outcome following anatomic lung resections: an analysis from the European Society of Thoracic Surgeons database. Eur J Cardiothorac Surg 2017;51:490-7.

30. Seo YS, Kim HJ, Wu HG, et al. Lobectomy versus stereotactic ablative radiotherapy for medically operable patients with stage IA non-small cell lung cancer: A virtual randomized phase III trial stratified by age. Thorac Cancer 
2019;10:1489-99.

31. Pardolesi A, Veronesi G, Solli P, et al. Use of indocyanine green to facilitate intersegmental plane identification during robotic anatomic segmentectomy. J Thorac Cardiovasc Surg 2014;148:737-8.

32. Wilson JL, Louie BE, Cerfolio RJ, et al. The prevalence of nodal upstaging during robotic lung resection in early stage non-small cell lung cancer. Ann Thorac Surg 2014;97:1901-6; discussion 1906-7.

33. Beckert AK, Huisingh-Scheetz M, Thompson K, et al.

Cite this article as: Veronesi G, Bruschini P, Novellis P. Robotic surgery can extend surgical indication in patients with lung cancer and impaired function. J Thorac Dis 2019;11(11):E224-E228. doi: 10.21037/jtd.2019.10.14
Screening for Frailty in Thoracic Surgical Patients. Ann Thorac Surg 2017;103:956-61.

34. Boujibar F, Bonnevie T, Debeaumont D, et al. Impact of prehabilitation on morbidity and mortality after pulmonary lobectomy by minimally invasive surgery: a cohort study. J Thorac Dis 2018;10:2240-8.

35. Osarogiagbon RU, Veronesi G, Fang W, et al. Early-Stage NSCLC: Advances in Thoracic Oncology 2018. J Thorac Oncol 2019;14:968-78. 